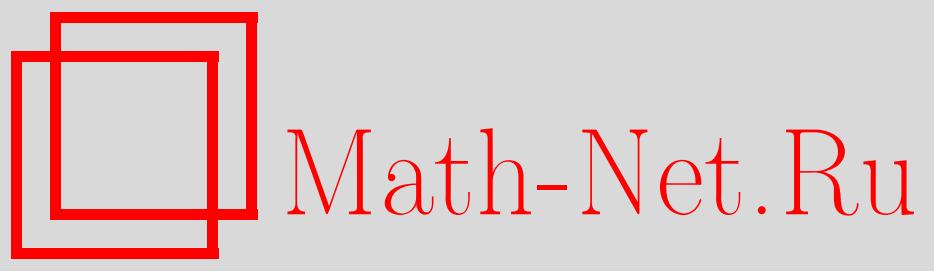

М. А. Соловьев, Обобщенное соответствие Вейля и алгебры мойаловских мультипликаторов, ТМФ, 2012, том 173, номер 1, 38-59

DOI: https://doi.org/10.4213/tmf8332

Использование Общероссийского математического портала Math-Net.Ru подразумевает, что вы прочитали и согласны с пользовательским соглашением http://www.mathnet.ru/rus/agreement

Параметры загрузки:

IP: 18.234 .197 .8

26 апреля 2023 г., 15:21:09

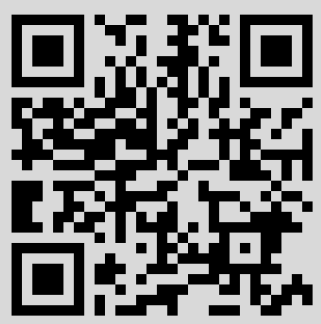




\title{
ОБОБЩЕННОЕ СООТВЕТСТВИЕ ВЕЙЛЯ И АЛГЕБРЫ МОЙАЛОВСКИХ МУЛЬТИПЛИКАТОРОВ
}

\begin{abstract}
Показано, что соответствие Вейля и понятие мультипликатора Мойала могут быть естественным образом распространены на классы обобщенных функций, более широкие, чем распределения умеренного роста. Это обобщение мотивировано возможными приложениями к некоммутативной квантовой теории поля. Доказано, что при разумных ограничениях на пространство пробных функций $E \subset L^{2}$ каждый оператор в $L^{2}$, определенный на $E$ и непрерывный в топологиях $E$ и $L^{2}$, имеет вейлевский символ, который определен как обобщенная функция на подвергнутом преобразованию Вигнера-Мойала тензорном квадрате пространства $E$. Дана точная характеризация преобразований Вейля мойаловских мультипликаторов пространств Гельфанда-Шилова $S_{\beta}^{\beta}$.
\end{abstract}

Ключевые слова: вейлевские символы, звездочное произведение, преобразование Вигнера-Мойала, группа Вейля-Гейзенберга, некоммутативная теория поля, топологические *-алгебры, обобщенные функции.

\section{1. ВВЕДЕНИЕ}

Соответствие Вейля [1] и звездочное произведение Мойала [2] играют центральную роль в квантовой механике на фазовом пространстве [3], и исчисление вейлевских символов находит все новые применения в различных областях исследования. В физической литературе вейлевское квантование излагается, как правило, на эвристическом уровне, однако уже довольно давно развит соответствующий строгий формализм, опирающийся на теорию распределений умеренного роста (см., например, [4]). В настоящей статье обсуждается его обобщение на более широкие классы распределений. Такое обобщение представляется целесообразным в связи с интенсивным развитием в последние годы квантовой теории поля на некоммутативных пространствах, нацеленной на изучение взаимоотношения квантовых явлений и гравитации на планковских пространственно-временных масштабах [5]. Модели некоммутативной теории поля конструируются заменой обычных произведений полей в функционале действия звездочными произведениями и обычно изучаются с помощью теории возмущений (см. обзор [6]). Однако есть доводы [7] в пользу того,

* Физический институт им. П. Н. Лебедева РАН, Москва, Россия.

E-mail: soloviev@lpi.ru 
что рамки теории распределений умеренного роста слишком узки для последовательной формулировки общих принципов квантовой теории поля на некоммутативном пространстве-времени. Одна из основных проблем состоит в правильной формулировке условия причинности, поскольку звездочное произведение нелокально и его разложение по степеням параметра некоммутативности сходится лишь на аналитических функциях. Пробные функции такого типа использовались ранее в нелокальной квантовой теории поля и, в частности, в работе [8] при обобщении вайтмановского аксиоматического подхода [9] на нелокальные поля. В работах [10], [11] показано, что пространства $S_{\alpha}^{\beta}$, введенные Гельфандом и Шиловым [12] и использовавшиеся в работе [8], являются топологическими алгебрами относительно произведения Мойала, если $\alpha \geqslant \beta$. Это позволяет определить соответствующие им алгебры мойаловских мультипликаторов по дуальности аналогично тому, как такая алгебра определяется [13]-[15] для пространства Шварца $S$, являющегося пространством пробных функций для распределений умеренного роста. Свойства этих алгебр изучались в работах [16], [17]. Обобщенное преобразование Вейля, рассматриваемое в настоящей статье, дает их операторную реализацию.

Произведение Мойала функций $f(x)$ и $g(x)$ на линейном симплектическом пространстве является некоммутативной деформацией обычного произведения, порождаемой симплектической структурой. Если билинейная симплектическая форма на четномерном пространстве $\mathbb{R}^{d}$ задается антисимметричной матрицей $\theta$, то

$$
\left(f \star_{\theta} g\right)(x) \stackrel{\text { def }}{=} \frac{1}{\pi^{d} \operatorname{det} \theta} \iint f(x+z) g\left(x+z^{\prime}\right) e^{-2 i z \cdot \theta^{-1} z^{\prime}} d z d z^{\prime} .
$$

Произведение (1) корректно определено для всех быстро убывающих гладких функций, составляющих пространство Шварца $S\left(\mathbb{R}^{d}\right)$, и нетрудно проверить, что $S\left(\mathbb{R}^{d}\right)$ с этим произведением является ассоциативной топологической алгеброй. Считая, что $f, g \in S\left(\mathbb{R}^{d}\right)$, и используя обычные обозначения для преобразования Фурье

$$
(\mathcal{F} f)(\zeta)=\hat{f}(\zeta)=\frac{1}{(2 \pi)^{d / 2}} \int f(x) e^{-i x \cdot \zeta} d x
$$

мы можем записать (1) как

$$
\left(f \star_{\theta} g\right)(x)=\frac{1}{(2 \pi)^{d}} \int f\left(x-\frac{1}{2} \theta \zeta\right) \hat{g}(\zeta) e^{i x \cdot \zeta} d \zeta=\frac{1}{(2 \pi)^{d}} \int \hat{f}(\zeta) g\left(x+\frac{1}{2} \theta \zeta\right) e^{i x \cdot \zeta} d \zeta
$$

В такой записи ясно, что $f \star_{\theta} g \rightarrow f \cdot g$ при $\theta \rightarrow 0$. Применяя к (2) оператор Фурье, мы получаем

$$
\widehat{f \star_{\theta} g}=\hat{f} \circledast_{\theta} \hat{g}
$$

где

$$
\begin{gathered}
\left(\hat{f} \circledast_{\theta} \hat{g}\right)(\zeta) \stackrel{\text { def }}{=} \frac{1}{(2 \pi)^{d / 2}} \int \hat{f}\left(\zeta-\zeta^{\prime}\right) \hat{g}\left(\zeta^{\prime}\right) e^{-(i / 2) \zeta \theta \zeta^{\prime}} d \zeta^{\prime}= \\
=\frac{1}{(2 \pi)^{d / 2}} \int \hat{f}\left(\zeta^{\prime}\right) \hat{g}\left(\zeta-\zeta^{\prime}\right) e^{(i / 2) \zeta \theta \zeta^{\prime}} d \zeta^{\prime}
\end{gathered}
$$


Функция (4) называется скрученной сверткой функций $f$ и $g$ и является некоммутативной деформацией обычной свертки. Удобство выбора коэффициентов в определении преобразования Фурье и в соотношении (4) станет ясным в разделе 5. Мнемоническое правило состоит в том, что мера Лебега в $\mathbb{R}^{d}$ в обоих случаях нормируется множителем $(2 \pi)^{-d / 2}$, что делает, в частности, оператор $\mathcal{F}$ унитарным. Поскольку матрица $\theta$ антисимметрична, из (2) вытекает, что

$$
\int\left(f \star_{\theta} g\right)(x) d x=\int f(x) g(x) d x \quad \text { при всех } \quad f, g \in S .
$$

Это свойство произведения Мойала играет важную роль в дальнейшем. Отметим, что в тех случаях, когда это не может привести к недоразумениям, мы будем использовать ниже обозначения $\star$ и $\circledast$ вместо $\star_{\theta}$ и $\circledast_{\theta}$.

Статья организована следующим образом. В разделе 2 понятие мойаловского мультипликатора [13]-[15] распространяется на любое пространство пробных функций $E$, являющееся подалгеброй алгебры $(S, \star)$. Показано, что множества $\mathcal{M}_{\theta, L}(E)$ и $\mathcal{M}_{\theta, R}(E)$ левых и правых мойаловских мультипликаторов для $E$ являются ассоциативными алгебрами с единицей. В разделе 3 показана важная роль существования в алгебре $E$ аппроксимативной единицы. Если $E$ ее имеет, мойаловские мультипликаторы можно аппроксимировать операторами $\star$-умножения на элементы $E$, что позволяет, в частности, определить инволютивную алгебру двусторонних мультипликаторов $\mathcal{M}_{\theta}(E)$. Объясняется связь этого определения с определением двойных централизаторов, используемым в теории $C^{*}$-алгебр [18]. В разделе 4 установлены легко проверяемые и достаточно общие условия, при которых структура алгебры относительно обычного умножения в $E$ допускает непрерывную деформацию в структуру алгебры относительно ћ-произведения. В разделе 5 мы напоминаем определения соответствия Вейля и преобразования Вигнера-Мойала, излагая их в удобной для дальнейшего форме и подчеркивая их связь с группой Вейля-Гейзенберга. В разделе 6 описывается общая схема распространения соответствия Вейля на более широкие, чем распределения Шварца, классы обобщенных функций. Указаны естественные с точки зрения функционального анализа ограничения на пространство пробных функций, при которых исчисление вейлевских символов допускает такое обобщение. Доказано, что при этих ограничениях существует единственное продолжение преобразования Вейля по непрерывности, которое устанавливает изоморфизм между множеством непрерывных операторов из $E$ в сопряженное пространство $E^{\prime}$ и множеством обобщенных функций, определенных на проективном тензорном произведении $E \widetilde{\otimes}_{\pi} E$, подвергнутом преобразованию Вигнера-Мойала. Как следствие, каждый оператор в гильбертовом пространстве $L^{2}$ с областью определения $E$, непрерывный относительно топологий $E$ и $L^{2}$, имеет вейлевский символ в этом классе обобщенных функций. Построение иллюстрируется на примере пространств $S_{\beta}^{\beta}$. В разделе 7 доказано, что обобщенное соответствие Вейля устанавливает изоморфизм между алгеброй $\mathcal{M}_{\theta, L}\left(S_{\beta}^{\beta}\right)$ и алгеброй операторов в $L^{2}$, непрерывно отображающих $S_{\beta}^{\beta}$ в себя. Раздел 8 посвящен заключительным замечаниям. В приложении доказана лемма, устанавливающая простые достаточные условия существования в звездочной алгебре аппроксимативной единицы. 


\section{2. МОЙАЛОВСКИЕ МУЛЬТИПЛИКАТОРЫ}

Пусть $E$ - локально выпуклое пространство, т. е. топологическое векторное пространство, имеющее базис выпуклых окрестностей начала. Если оно является линейным подпространством другого топологического векторного пространства $E_{0}$ и тождественное отображение $E \rightarrow E_{0}$ непрерывно, мы говорим, что $E$ - локально выпуклое подпространство в $E_{0}$. Если при этом $E_{0}$ - алгебра, а $E$ - ее подалгебра с совместно непрерывным умножением, то мы говорим, что $E$ является локально выпуклой подалгеброй алгебры $E_{0}$.

Пусть $E$ - плотное локально выпуклое подпространство пространства Шварца $S$. Тогда мы имеем цепочку канонических непрерывных вложений

$$
E \rightarrow S \rightarrow S^{\prime} \rightarrow E^{\prime}
$$

где штрих используется для обозначения сопряженного пространства, снабженного сильной топологией, т. е. топологией равномерной сходимости на ограниченных множествах. Последнее отображение в (6) сопряжено первому, и в силу элементарных свойств сопряженных отображений ([19], § IV.2.3) его образ плотен в $E^{\prime}$ в слабой топологии $\sigma\left(E^{\prime}, E\right)$, а если $E$ полурефлексивно, то $S^{\prime}$ сильно плотно в $E^{\prime}$, поскольку замыкание векторного пространства одно и то же для всех локально выпуклых топологий, согласующихся с двойственностью ([19], § IV.3.1). Значение линейной формы $u \in E^{\prime}$ на функции $f \in E$ мы обозначаем, как обычно, через $\langle u, f\rangle$. Если $E$ является топологической алгеброй относительно звездочного произведения (1), то можно определить произведения $u \star f$ и $f \star u$, где $u \in E^{\prime}$ и $f \in E$, посредством формул

$$
\langle u \star f, g\rangle=\langle u, f \star g\rangle, \quad\langle f \star u, g\rangle=\langle u, g \star f\rangle, \quad g \in E .
$$

Поскольку здесь правые части линейны и непрерывны по $g \in E$, данные произведения корректно определены как элементы $E^{\prime}$, и они расширяют $\star$-умножение в пространстве $S$. Действительно, если $u \in S$, то в силу соотношения (5) и ассоциативности алгебры $(S, \star)$ мы имеем цепочку равенств

$$
\langle u \star f, g\rangle=\int(u \star f)(x) g(x) d x=\int((u \star f) \star g)(x) d x=\int(u \star(f \star g))(x) d x=\langle u, f \star g\rangle
$$

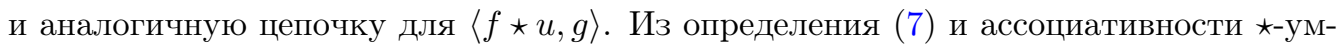
ножения в $E$ прямо следует, что

$$
(u \star f) \star g=u \star(f \star g), \quad(f \star u) \star g=f \star(u \star g), \quad f \star(g \star u)=(f \star g) \star u
$$

для всех $u \in E^{\prime}$ и $f, g \in E$. Линейные отображения $u \rightarrow u \star f$ и $u \rightarrow f \star u$ сопряжены к непрерывным отображениям $g \rightarrow f \star g$ и $g \rightarrow g \star f$ соответственно, и поэтому они непрерывны как относительно слабой, так и относительно сильной топологии пространства $E^{\prime}$. Поскольку $E$ плотно в $E^{\prime}$, не существует никаких других непре-

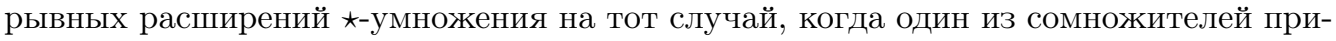
надлежит $E^{\prime}$. Отображения $f \rightarrow u \star f$ и $f \rightarrow f \star u$ из $E$ в $E^{\prime}$ также непрерывны. Рассмотрим, например, первое из них. Для любого $\epsilon>0$ существует окрестность нуля $W$ в $E$ такая, что функционал $u$ ограничен на $W$ числом $\epsilon$. Поскольку отображение $(f, g) \rightarrow f \star g$ совместно непрерывно, найдутся окрестности $U$ и $V$ такие, 
что $f \star g \in W$ при всех $f \in U$ и $g \in V$. Для любого ограниченного множества $Q \subset E$ найдется $\delta>0$ такое, что $\delta Q \subset V$ и, значит, $\sup _{g \in Q}|\langle u, f \star g\rangle| \leqslant \epsilon$ при всех $f \in \delta U$, что и доказывает непрерывность отображения $f \rightarrow u \star f$.

ОПредЕЛЕНИЕ 1 . Пусть $E$ - локально выпуклая подалгебра алгебры $\left(S, \star_{\theta}\right)$. Лe-

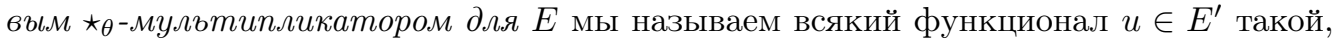
что $u \star_{\theta} f \in E$ при всех $f \in E$ и отображение $E \rightarrow E: f \rightarrow u \star_{\theta} f$ непрерывно. Множество всех левых мойаловских мультипликаторов для $E$ обозначаем через $\mathcal{M}_{\theta, L}(E)$. Аналогично определяется множество правых $\star_{\theta}$-мультипликаторов $\mathcal{M}_{\theta, R}(E)$.

Отметим, что если к $E$ применима теорема о замкнутом графике, например если $E$ - пространство Фреше, то непрерывность отображения $f \rightarrow u \star f$ соблюдается автоматически, поскольку его график замкнут. Действительно, пусть $f_{\gamma} \rightarrow f$ и $u \star$ $f_{\gamma} \rightarrow h$, тогда для любой функции $g \in E$ мы имеем

$$
\langle h, g\rangle=\lim _{\gamma}\left\langle u \star f_{\gamma}, g\right\rangle=\lim _{\gamma}\left\langle u, f_{\gamma} \star g\right\rangle=\langle u, f \star g\rangle=\langle u \star f, g\rangle
$$

и, значит, $h=u \star f$. По дуальности корректно определены произведения мойаловских мультипликаторов с элементами $w \in E^{\prime}$, а именно:

$$
\langle w \star u, f\rangle:=(w, u \star f), \quad\langle v \star w, f\rangle:=\langle w, f \star v\rangle, \quad u \in \mathcal{M}_{\theta, L}(E), \quad v \in \mathcal{M}_{\theta, R}(E) .
$$

Таким образом, левые (правые) мультипликаторы для $E$ служат правыми (левыми) мультипликаторами для $E^{\prime}$. Если и к $E^{\prime}$ применима теорема о замкнутом графике, то при фиксированных $u$ и $v$ отображения $w \rightarrow w \star u$ и $w \rightarrow v \star w$ из $E^{\prime}$ в $E^{\prime}$ непрерывны. Очевидно, $E$ содержится как в $\mathcal{M}_{\theta, L}(E)$, так и в $\mathcal{M}_{\theta, R}(E)$, и операции (10) расширяют операции (7).

ПрЕДЛОЖЕНИЕ 1. Пространства $\mathcal{M}_{\theta, L}(E)$ и $\mathcal{M}_{\theta, R}(E)$ являются униталъными ассочиативными алгебрами относительно $\star_{\theta}$-произведения.

Доказательство. Покажем сначала, что $u_{1}, u_{2} \in \mathcal{M}_{\theta, L}(E) \Rightarrow u_{1} \star u_{2} \in \mathcal{M}_{\theta, L}(E)$. Пусть $f, g \in E$. Используя последовательно (7), (10), (9) и снова (7), мы получаем

$$
\begin{aligned}
\left\langle\left(u_{1} \star u_{2}\right) \star f, g\right\rangle & =\left\langle u_{1} \star u_{2}, f \star g\right\rangle=\left\langle u_{1}, u_{2} \star(f \star g)\right\rangle= \\
& =\left\langle u_{1},\left(u_{2} \star f\right) \star g\right\rangle=\left\langle u_{1} \star\left(u_{2} \star f\right), g\right\rangle .
\end{aligned}
$$

Значит, $\left(u_{1} \star u_{2}\right) \star f=u_{1} \star\left(u_{2} \star f\right) \in E$, причем отображение $f \rightarrow\left(u_{1} \star u_{2}\right) \star f$ непрерывно. Тем самым $u_{1} \star u_{2} \in \mathcal{M}_{\theta, L}(E)$. Аналогичным образом проверяется, что $v_{1}, v_{2} \in \mathcal{M}_{\theta, R}(E) \Rightarrow v_{1} \star v_{2} \in \mathcal{M}_{\theta, R}(E)$. Далее,

$\left\langle\left(u_{1} \star u_{2}\right) \star u_{3}, f\right\rangle=\left\langle u_{1} \star u_{2}, u_{3} \star f\right\rangle=\left\langle u_{1}, u_{2} \star\left(u_{3} \star f\right)\right\rangle=\left\langle u_{1},\left(u_{2} \star u_{3}\right) \star f\right\rangle=\left\langle u_{1} \star\left(u_{2} \star u_{3}\right), f\right\rangle$,

что доказывает ассоциативность алгебры $\mathcal{M}_{\theta, L}(E)$. Обозначим через $\mathbb{1}$ функционал $f \rightarrow \int f(x) d x$, задаваемый функцией, тождественно равной единице. Из (5) следует, что

$$
\langle\mathbb{1} \star f, g\rangle=\langle f \star \mathbb{1}, g\rangle=\int f(x) g(x) d x \quad \text { при всех } \quad f, g \in E .
$$

Значит, $\mathbb{1} \star f=f \star \mathbb{1}=f$, и тем самым $\mathbb{1}$ принадлежит как $\mathcal{M}_{\theta, L}(E)$, так и $\mathcal{M}_{\theta, R}(E)$ и служит единицей этих алгебр. Предложение доказано. 
Рассмотренному расширению мойаловского умножения на элементы $E^{\prime}$ соответствует расширение операции скрученной свертки в фурье-двойственном пространстве. Пространство преобразований Фурье функций из $E$ мы обозначаем через $\widehat{E}$ и наделяем $\widehat{E}$ топологией, перенесенной из $E$. Если $g \in \widehat{E}, v \in \widehat{E}^{\prime}$, то

$$
v \circledast g=\mathcal{F}\left(\mathcal{F}^{-1} v \star \mathcal{F}^{-1} g\right), \quad g \circledast v=\mathcal{F}\left(\mathcal{F}^{-1} g \star \mathcal{F}^{-1} v\right) .
$$

Это единственное возможное расширение произведения (4) по непрерывности на $\widehat{E}^{\prime} \times \widehat{E}$ и на $\widehat{E} \times \widehat{E}^{\prime}$.

\section{3. АППРОКСИМАТИВНАЯ ЕДИНИЦА И ДВОЙНЫЕ ЦЕНТРАЛИЗАТОРЫ}

В общей теории топологических алгебр мультипликаторы определяются иначе, чем в предыдущем разделе, однако нетрудно видеть, что эти определения равносильны при наличии в алгебре аппроксимативной единицы. Для любого топологического векторного пространства $E$ мы обозначаем через $\mathcal{L}(E)$ пространство линейных непрерывных отображений из $E$ в $E$, снабженное топологией равномерной сходимости на ограниченных множествах. Если $A$ - топологическая алгебра, ее аппроксимативной единицей называют последовательность ${ }^{1)}$ элементов $e_{n} \in A$ такую, что $e_{n} a \rightarrow a$ и $a e_{n} \rightarrow a$ для всех $a \in A$. Левым мультипликатором алгебры $A$ называют всякий оператор $L \in \mathcal{L}(A)$, для которого при всех $a, b \in A$

$$
L(a b)=L(a) b .
$$

Множество левых мультипликаторов мы обозначим через $\mathscr{M}_{L}(A)$. Ясно, что оно является замкнутой подалгеброй алгебры $\mathcal{L}(A)$ и имеется естественное вложение $A \rightarrow \mathscr{M}_{L}(A)$. Аналогичным образом определяется алгебра правых мультипликаторов $\mathscr{M}_{R}(A)$, с заменой соотношения (12) на

$$
R(a b)=a R(b) .
$$

Следуя [18], двойным централизатором алгебры $A$ мы называем пару операторов $(L, R)$ со свойствами $(12),(13)$, связанных соотношением

$$
R(a) b=a L(b)
$$

Легко проверить, что множество двойных централизаторов $\mathscr{M}(A)$ есть замкнутое подпространство в $\mathcal{L}(A) \oplus \mathcal{L}(A)$ и оно является алгеброй относительно умножения по правилу

$$
\left(L_{1}, R_{1}\right)\left(L_{2}, R_{2}\right)=\left(L_{1} L_{2}, R_{2} R_{1}\right) .
$$

Большинство пространств, используемых в теории обобщенных функций, являются монтелевскими. Для таких пространств, имеющих структуру инволютивной топологической алгебры, можно указать легко проверяемые достаточные условия существования аппроксимативной единицы, которые сформулированы ниже в приложении в виде леммы. В работе [16] доказано, что алгебры $\left(S_{\alpha}^{\beta}, \star\right), \alpha \geqslant \beta$, имеют секвенциальную аппроксимативную единицу.

1) В более общем определении используется понятие сети элементов, но мы будем рассматривать лишь секвенциальные аппроксимативные единицы. 
ПредлОЖЕНИЕ 2. Пусть $E$ - локально выпуклая подалгебра алгебры $\left(S, \star_{\theta}\right)$. Если в $E$ существует аппроксимативная единища, то алгебра $\mathcal{M}_{\theta, L}(E)$ канонически изоморфна алгебре $\mathscr{M}_{L}(E)$. Если, кроме того, пространство $Е$ монтелевское, то $\mathscr{M}_{L}(E)$ совпадает с замыканием в $\mathcal{L}(E)$ множества операторов левого $\star_{\theta}$-умножения на элементы $E$. Аналогичные утверждения верны для алгебр $\mathcal{M}_{\theta, R}(E)$ u $\mathscr{M}_{R}(E)$.

Доказательство. Пусть $u \in \mathcal{M}_{\theta, L}(E)$. Обозначим через $L_{u}$ оператор $f \rightarrow u \star f$. Первое из соотношений (9) показывает, что $L_{u} \in \mathscr{M}_{L}(E)$. Из существования в $E$ аппроксимативной единицы вытекает, в частности, что множество $\{f \star g: f, g \in E\}$ плотно в $E$, поэтому гомоморфизм алгебр

$$
\mathcal{M}_{\theta, L}(E) \rightarrow \mathcal{L}(E): u \rightarrow L_{u}
$$

инъективен. С другой стороны, всякий оператор $V \in \mathcal{L}(E)$ определяет функционал $v \in E^{\prime}$ по формуле

$$
\langle v, f\rangle=\int V(f) d x
$$

причем отображение $V \rightarrow v$ из $\mathcal{L}(E)$ в $E^{\prime}$ непрерывно по определению топологии этих пространств. Если $V \in \mathscr{M}_{L}(E)$, то в силу (5) мы имеем

$$
\langle v, f \star g\rangle=\int V(f \star g) d x=\int V(f) g d x .
$$

Значит, $v \in \mathcal{M}_{\theta, L}(E)$ и $L_{v}=V$. Таким образом, соотношение (16) есть изоморфизм алгебр $\mathcal{M}_{\theta, L}(E)$ и $\mathscr{M}_{L}(E)$. Далее, если $\left\{e_{n}\right\}$ - аппроксимативная единица алгебры $E$ и $L \in \mathscr{M}_{L}(E)$, то из соотношения $L\left(e_{n} \star f\right)=L\left(e_{n}\right) \star f$ следует, что последователь-

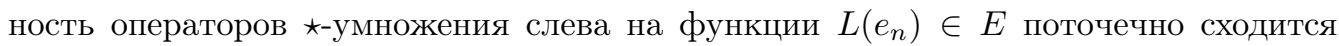
к $L$. Если пространство $E$ монтелевское, к нему применима обобщенная теорема Банаха-Штейнгауза ([19], § III.4.6), согласно которой отсюда следует, что последовательность $L\left(e_{n}\right) \star$ сходится к $L$ равномерно на каждом предкомпактном подмножестве в $E$. Поскольку в монтелевском пространстве любое ограниченное множество предкомпактно, мы заключаем, что $L\left(e_{n}\right) \star \rightarrow L$ по топологии пространства $\mathcal{L}(E)$.

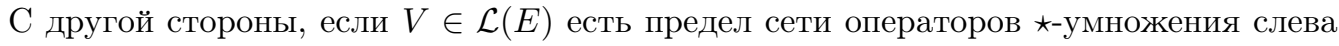
на функции $h_{\gamma} \in E$, то

$$
V(f \star g)=\lim _{\gamma} h_{\gamma} \star(f \star g)=\lim _{\gamma}\left(h_{\gamma} \star f\right) \star g=V(f) \star g
$$

и тем самым $V \in \mathscr{M}_{L}(E)$. Случай правых мультипликаторов рассматривается аналогично, что завершает доказательство.

Предложение 2 показывает, что естественной топологией алгебр $\mathcal{M}_{\theta, L}(E)$ и $\mathcal{M}_{\theta, R}(E)$ является та, которая индуцируется из $\mathcal{L}(E)$. Если $E$ наряду с каждой функцией $f$ содержит комплексно-сопряженную функцию $f^{*}$ и является инволютивной алгеброй, то для элементов $u \in E^{\prime}$ инволюция определяется посредством формулы $\left\langle u^{*}, f\right\rangle=\left\langle u, f^{*}\right\rangle^{*}$. Отображение $u \rightarrow u^{*}$ есть антилинейный изоморфизм между $\mathcal{M}_{\theta, L}(E)$ и $\mathcal{M}_{\theta, R}(E)$, поскольку $(u \star f)^{*}=f^{*} \star u^{*}$. Теперь мы определим 
аналог инволютивной алгебры мойаловских мультипликаторов, введенной Антонецем [13] для пространства Шварца и изучавшейся в работах [14], [15]. Рассмотрим пересечение

$$
\mathcal{M}_{\theta}(E)=\mathcal{M}_{\theta, L}(E) \cap \mathcal{M}_{\theta, R}(E) .
$$

ПРЕДЛОЖЕНИЕ 3. Если Е- инволютивная локально выпуклая подалгебра алгебpъь $\left(S, \star_{\theta}\right)$ с аппроксимативной единицей $\left\{e_{n}\right\}$, то $\mathcal{M}_{\theta}(E)$ является инволютивной унитальной алгеброй, канонически изоморбной алгебре двойных централизаторов $\mathscr{M}(E)$. Если, кроме того, пространство $Е$ монтелевское, то $Е$ плотно в $\mathcal{M}_{\theta}(E)$.

ДоказАтЕЛьство. Каждый элемент $u \in \mathcal{M}_{\theta}(E)$ порождает операторы $L_{u}=u \star$ и $R_{u}=\star u$, которые в силу первого и третьего из соотношений (9) принадлежат $\mathscr{M}_{L}(E)$ и $\mathscr{M}_{R}(E)$ соответственно. Из второго из соотношений (9) следует, что

$$
R_{u}(f) \star g=f \star L_{u}(g) .
$$

Значит, $\left(L_{u}, R_{u}\right) \in \mathscr{M}(E)$. Ясно также, что отображение $\mathcal{M}_{\theta}(E) \rightarrow \mathscr{M}(E): u \rightarrow$ $\left(L_{u}, R_{u}\right)$ линейно и инъективно. Обратно, пусть $(L, R)$ - двойной централизатор алгебры $E$ и $\langle v, f\rangle=\int L(f) d x,\left\langle v^{\prime}, f\right\rangle=\int R(f) d x$. Используя (5) и (18), получаем

$$
\langle v, f \star g\rangle=\int L(f) g d x=\int f R(g) d x=\left\langle v^{\prime}, f \star g\right\rangle .
$$

Следовательно, $v=v^{\prime}$. Таким образом, отображение $\mathcal{M}_{\theta}(E) \rightarrow \mathscr{M}(E)$ биективно. Умножение в $\mathcal{M}_{\theta}(E)$ можно определить любой из двух формул

$$
\langle u \star v, f\rangle=\langle u, v \star f\rangle, \quad\langle u \star v, f\rangle=\langle v, f \star u\rangle .
$$

Действительно, если заменить $f$ на $e_{n} \star f$, то в силу формул (9) правые части в (19) могут быть записаны как $\int(f \star u)\left(v \star e_{n}\right) d x$. Переходя к пределу $n \rightarrow \infty$ и учитывая непрерывность отображений $f \rightarrow v \star f$ и $f \rightarrow f \star u$, мы видим, что эти определения эквивалентны. Ясно также, что при отождествлении $\mathcal{M}_{\theta}(E)$ с $\mathscr{M}(E)$ они переходят в определение (15). Топология, индуцированная на $\mathcal{M}_{\theta}(E)$ из $\mathcal{L}(E) \oplus \mathcal{L}(E)$, совпадает с верхней гранью топологий, индуцированных вложениями $\mathcal{M}_{\theta}(E) \rightarrow \mathcal{M}_{\theta, L}(E)$ и $\mathcal{M}_{\theta}(E) \rightarrow \mathcal{M}_{\theta, R}(E)$. Если $u \in \mathcal{M}_{\theta}(E)$, то $\left(u \star e_{n}\right) \star f \rightarrow u \star f$ и $f \star\left(u \star e_{n}\right) \rightarrow f \star u$ при любой $f \in E$. Как было отмечено при доказательстве предложения 2 , если пространство $E$ монтелевское, отсюда следует, что $\left(u \star e_{n}\right) \star \rightarrow u \star и \star\left(u \star e_{n}\right) \rightarrow \star u$ по топологии $\mathcal{L}(E)$. Предложение доказано.

\section{4. НЕПРЕРЫВНАЯ ДЕФОРМАЦИЯ ПОТОЧЕЧНОГО ПРОИЗВЕДЕНИЯ В ЗВЕЗДОЧНОЕ}

В работе [11] установлено, что при условии $\alpha \geqslant \beta$ обычное произведение функций в пространстве $S_{\alpha}^{\beta}$ может быть непрерывно деформировано в скрученное произведение (1). Здесь мы докажем несколько более общую теорему, но прежде введем обозначения. Для любого локально выпуклого пространства $E$ обозначим через $E \widetilde{\otimes} E$ пополненное проективное тензорное произведение двух экземпляров $E$. Знаменитая теорема Шварца о ядре может быть записана в виде равенства

$$
S\left(\mathbb{R}^{d}\right) \widetilde{\otimes} S\left(\mathbb{R}^{d}\right)=S\left(\mathbb{R}^{2 d}\right) .
$$


Если $E$ - полное ядерное подпространство в $S\left(\mathbb{R}^{d}\right)$, то $E \widetilde{\otimes} E$ является полным ядерным подпространством в $S\left(\mathbb{R}^{2 d}\right)$ (см. [17], предложение 3). При этом $\mathcal{F}(E \widetilde{\otimes} E)=$ $\widehat{E} \widetilde{\otimes} \widehat{E}$.

Теорема 1. Пусть $E$ - полное ядерное подпространство в $S\left(\mathbb{R}^{d}\right)$ и топологическая алгебра относительно поточечного умножения. Пусть $\theta$ - симплектическая форма на $\mathbb{R}^{d}$. Предположим, что функиия $e^{-(i / 2) \theta}$ является поточечным мультипликатором для $\widehat{E} \widetilde{\otimes} \widehat{E}$. Тогда Е является топологической алгеброй относительно произведения Мойала (1), а $\widehat{E}$ - топологической алгеброй относительно скрученной свертки (4). Если, кроме того, пространство $\widehat{E} \widetilde{\otimes} \widehat{E}$ монтелевское и для всякой функиии $h \in \widehat{E} \widetilde{\otimes} \widehat{E}$ множество $\left\{h e^{-(i / 2) t \theta}: 0 \leqslant t \leqslant 1\right\}$ в нем ограничено, то поточечное произведение элементов $E$ допускает непрерывную дебормацию в мойаловское произведение.

ДокАЗАТЕЛЬСтво. Из формул (3) и (4) следует, что

$$
\left(f \star_{\theta} g\right)(x)=\frac{1}{(2 \pi)^{d}} \iint \hat{f}(\zeta) g\left(\zeta^{\prime}\right) e^{-(i / 2) \zeta \cdot \theta \zeta^{\prime}+i x\left(\zeta+\zeta^{\prime}\right)} d \zeta d \zeta^{\prime}, \quad f, g \in E .
$$

Поэтому отображение $(f, g) \rightarrow f \star_{\theta} g$ можно рассматривать как композицию следующих пяти отображений:

$$
E \times E \stackrel{\otimes}{\longrightarrow} E \widetilde{\otimes} E \stackrel{\mathcal{F}}{\longrightarrow} \widehat{E} \widetilde{\otimes} \widehat{E} \cdot \stackrel{e^{-(i / 2) \theta}}{\longrightarrow} \widehat{E} \widetilde{\otimes} \widehat{E} \stackrel{\mathcal{F}^{-1}}{\longrightarrow} E \widetilde{\otimes} E \stackrel{\mathbf{m}}{\longrightarrow} E,
$$

где через $\mathbf{m}$ обозначено сужение на диагональ $h(x, y) \rightarrow h(x, x)$. Первое, второе и четвертое из отображений (21) непрерывны по определению топологии участвующих пространств, а третье - в силу предположения о том, что $e^{-(i / 2) \theta}$ есть поточечный мультипликатор. По категорному смыслу проективного тензорного произведения каждому билинейному непрерывному отображению $E \times E \rightarrow E$ однозначно соответствует линейное непрерывное отображение $E \widetilde{\otimes} E \rightarrow E$. Ясно, что $\mathbf{m}$ является тем линейным отображением, которое соответствует умножению $(f, g) \rightarrow f \cdot g$, и его непрерывность вытекает из предположения, что $E$ есть топологическая алгебра по обычному умножению. Итак, все отображения в цепочке (21) непрерывны, и тем самым $E$ является топологической алгеброй относительно умножения $(f, g) \rightarrow f \star_{\theta} g$. Пусть теперь $\widehat{E} \widetilde{\otimes} \widehat{E}-$ монтелевское пространство и множество $\left\{(\hat{f} \otimes \hat{g}) e^{-(i / 2) t \theta}: 0 \leqslant t \leqslant 1\right\}$ ограничено. По определению монтелевского пространства [19] это множество относительно компактно. Поэтому для любой положительной последовательности $t_{\nu} \rightarrow 0$ соответствующая последовательность функций $(\hat{f} \otimes \hat{g}) e^{-(i / 2) t_{\nu} \theta}$ имеет по крайней мере одну предельную точку. Поскольку топология $\widehat{E} \widetilde{\otimes} \widehat{E}$ сильнее топологии поточечной сходимости, предельной точкой может быть только $\hat{f} \otimes \hat{g}$. Следовательно, $f \star_{t \theta} g \rightarrow f \cdot g$ по топологии $E$ при $t \rightarrow 0$. Более того, согласно обобщенной теореме Банаха-Штейнгауза ([19], § III.4.6) последовательность операторов умножения на функции $e^{-(i / 2) t_{\nu} \theta}$ сходится к единичному оператору равномерно на каждом предкомпактном множестве в $\widehat{E} \widetilde{\otimes} \widehat{E}$. Пусть $\mathfrak{S}-$ семейство всех ограниченных подмножеств $E$. Если $A, B \in \mathfrak{S}$, то множество функций $\hat{f} \otimes \hat{g}$, где $f \in A$ и $g \in B$, предкомпактно в $\widehat{E} \widetilde{\otimes} \widehat{E}$, и мы заключаем, что при $t \rightarrow 0$ билинейное отображение $(f, g) \rightarrow f \star_{t \theta} g$ стремится к поточечному умножению 
по топологии $\mathfrak{S} \times \mathfrak{S}$-сходимости в пространстве непрерывных билинейных отображений $E \times E \rightarrow E$. Аналогичные аргументы показывают, что билинейная операция $\star_{t \theta}$ непрерывна в любой другой точке $t \in[0,1]$. Теорема доказана.

\section{5. СООТВЕТСТВИЕ ВЕЙЛЯ И ПРЕОБРАЗОВАНИЕ ВИГНЕРА-МОЙАЛА}

Пусть теперь $\theta$ - стандартная симплектическая линейная структура на пространстве $\mathbb{R}^{2 n}=\mathbb{R}^{n} \times \mathbb{R}^{n}$, точки которого мы будем обозначать через $z=(q, p)$, как это принято в формулировке квантовой механики на фазовом пространстве. Тогда

$$
\theta\left(z, z^{\prime}\right)=z \cdot J z^{\prime}=q p^{\prime}-p q^{\prime}, \quad J=\left(\begin{array}{cc}
0 & I_{n} \\
-I_{n} & 0
\end{array}\right) .
$$

Через $L^{2}\left(\mathbb{R}^{n}, d q\right)$ обозначим гильбертово пространство квадратично-интегрируемых функций $\psi(q)$ и через $\mathfrak{q}_{j}$ и $\mathfrak{p}_{j}-$ операторы $\left(\mathfrak{q}_{j} \psi\right)(q)=q_{j} \psi(q)$ и $\left(\mathfrak{p}_{j} \psi\right)(q)=-i \hbar \partial_{j} \psi(q)$. Соответствие Вейля сопоставляет функции $f(q, p)$ на $\mathbb{R}^{2 n}$ оператор $\mathfrak{f}$ в $L^{2}\left(\mathbb{R}^{n}, d q\right)$ по правилу

$$
\begin{aligned}
f \longmapsto \mathfrak{f} & =\frac{1}{(2 \pi)^{2 n}} \int f(q, p) e^{i s(\mathfrak{q}-q)+i r(\mathfrak{p}-p)} d q d p d s d r= \\
& =\frac{1}{(2 \pi)^{n}} \int \hat{f}(s, r) e^{i s \mathfrak{q}+i r \mathfrak{p}} d s d r .
\end{aligned}
$$

Если функция $\hat{f}$ абсолютно интегрируема, то последний интеграл корректно определен, так как норма подынтегральной операторной функции мажорируется функцией $|\hat{f}(s, r)|$. Поскольку в основе лежит симплектическая структура, в ряде отношений предпочтительно пользоваться симплектическим преобразованием Фурье

$$
\tilde{f}(\zeta)=\left(\mathcal{F}_{J} f\right)(\zeta):=\frac{1}{(2 \pi)^{n}} \int f(z) e^{-i z \cdot J \zeta} d z=\frac{1}{(2 \pi)^{n}} \int f(q, p) e^{-i(\eta q-\xi p)} d q d p,
$$

где $\zeta=(\xi, \eta)$. Тогда определение (22) принимает вид

$$
f \longmapsto \mathfrak{f}=\frac{1}{(2 \pi)^{n}} \int \tilde{f}(\zeta) T_{\zeta}^{\hbar} d \zeta,
$$

где

$$
T_{\zeta}^{\hbar} \stackrel{\text { def }}{=} e^{i(\eta \mathfrak{q}-\xi \mathfrak{p})}=e^{-(i \hbar / 2) \eta \xi} e^{i \eta \mathfrak{q}} e^{-i \xi \mathfrak{p}} .
$$

Отметим, что при сделанном выборе коэффициентов симплектическое преобразование Фурье обратно самому себе, $\tilde{\tilde{f}}=f$, и связано с обычным преобразованием Фурье очевидной формулой

$$
\tilde{f}(\zeta)=\hat{f}(J \zeta)
$$

или, подробнее, $\tilde{f}(\xi, \eta)=\hat{f}(\eta,-\xi)$. Унитарные операторы $T_{\zeta}^{\hbar}$ Удовлетворяют соотношению

$$
T_{\zeta}^{\hbar} T_{\zeta^{\prime}}^{\hbar}=e^{-(i \hbar / 2) \zeta \cdot J \zeta^{\prime}} T_{\zeta+\zeta^{\prime}}^{\hbar}
$$


и реализуют проективное представление группы трансляций фазового пространства. Это представление линеаризуется на центральном расширении группы трансляций, называемом группой Вейля-Гейзенберга и обозначаемом через $G_{\mathrm{WH}}$. Группа $G_{\mathrm{WH}}$ состоит из элементов вида $(\alpha, \zeta)$ с законом умножения

$$
(\alpha, \zeta) \circ\left(\alpha^{\prime}, \zeta^{\prime}\right)=\left(\alpha+\alpha^{\prime}+\frac{1}{2} \zeta \cdot J \zeta^{\prime}, \zeta+\zeta^{\prime}\right)
$$

Из (27) следует, что операторы

$$
U_{\alpha, \zeta}^{\hbar}=e^{-i \hbar \alpha} T_{\zeta}^{\hbar}
$$

реализуют линейное унитарное представление группы $G_{\mathrm{WH}}$ в $L^{2}\left(\mathbb{R}^{n}, d q\right)$. Это представление неприводимо. Для любого другого унитарного неприводимого представления $U_{\alpha, \zeta}^{\prime}$ группы $G_{\mathrm{WH}}$ ограничение на ее центр имеет вид $U_{\alpha, 0}^{\prime}=e^{-i \lambda \alpha} I$ с некоторым вещественным $\lambda$ и по теореме Стоуна-фон Неймана $U_{\alpha, \zeta}^{\prime}$ унитарно эквивалентно представлению (28) в том (и, очевидно, только в том) случае, если $\lambda=\hbar$. Из формул $(23),(24)$ следует, что в явном виде действие оператора $\mathfrak{f}$ на функцию $\psi \in L^{2}\left(\mathbb{R}^{n}, d q\right)$ записывается как

$$
(\mathfrak{f} \psi)(x)=\frac{1}{(2 \pi \hbar)^{n}} \int f\left(\frac{1}{2}(x+q), p\right) e^{(i / \hbar) p(x-q)} \psi(q) d q d p .
$$

Соответственно, обозначая скалярное произведение в гильбертовом пространстве круглыми скобками, для матричных элементов оператора $\mathfrak{f}$ мы имеем представление

$$
(\varphi, \mathfrak{f}, \psi)=\int f(z) W_{\hbar}(\varphi, \psi)(z) d z
$$

где $W_{\hbar}(\varphi, \psi)$ - функция, называемая преобразованием Вигнера-Мойала пары $(\varphi, \psi)$ и определяемая выражением

$$
W_{\hbar}(\varphi, \psi)(q, p)=\frac{1}{(2 \pi \hbar)^{n}} \int \varphi^{*}\left(q-\frac{1}{2} x\right) \psi\left(q+\frac{1}{2} x\right) e^{-(i / \hbar) p x} d x
$$

Из формул $(23)$ и $(24)$ видно, что функцию $W_{\hbar}(\varphi, \psi)$ можно записать также в виде

$$
W_{\hbar}(\varphi, \psi)(z)=\frac{1}{(2 \pi)^{2 n}} \int\left(\varphi, T_{\zeta}^{\hbar} \psi\right) e^{i \zeta \cdot J z} d \zeta
$$

т. е. она связана с матричными элементами оператора $T_{\zeta}^{\hbar}$ вторым (с другим знаком в экспоненте) симплектическим преобразованием Фурье $\overline{\mathcal{F}}_{J}$.

В этом и следующих разделах операция скрученной свертки, порождаемая матрицей $\theta=\hbar J$, будет обозначаться через $\circledast \hbar$. Как было установлено впервые фон Нейманом (в предположении интегрируемости функций), скрученная свертка $\hat{f} \circledast \hbar \hat{g}$ соответствует произведению $\mathfrak{f g}$ преобразований Вейля функций $f$ и $g$. Это прямо следует из соотношения (27) и может рассматриваться как следствие того факта, что (28) есть представление группы $G_{\mathrm{WH}}$. Действительно, пусть

$$
\hat{h}(\zeta)=(\hat{f} \circledast \hbar \hat{g})(\zeta)=\frac{1}{(2 \pi)^{n}} \int \hat{f}\left(\zeta^{\prime}\right) \hat{g}\left(\zeta-\zeta^{\prime}\right) e^{(i \hbar / 2) \zeta \cdot J \zeta^{\prime}} d \zeta^{\prime},
$$


что в силу (26) равносильно равенству $\tilde{h}=\tilde{f} \circledast \hbar \tilde{g}$. Преобразование $(24)$ переводит функцию $h$ в оператор

$$
\begin{aligned}
\mathfrak{h} & =\frac{1}{(2 \pi)^{2 n}} \int \tilde{f}\left(\zeta^{\prime}\right) \tilde{g}\left(\zeta-\zeta^{\prime}\right) e^{(i \hbar / 2) \zeta \cdot J \zeta^{\prime}} T_{\zeta}^{\hbar} d \zeta^{\prime} d \zeta= \\
& =\frac{1}{(2 \pi)^{2 n}} \int \tilde{f}\left(\zeta^{\prime}\right) \tilde{g}\left(\zeta^{\prime \prime}\right) e^{(i \hbar / 2) \zeta^{\prime \prime} \cdot J \zeta^{\prime}} T_{\zeta^{\prime}+\zeta^{\prime \prime}}^{\hbar} d \zeta^{\prime} d \zeta^{\prime \prime}= \\
& =\frac{1}{(2 \pi)^{2 n}} \int \tilde{f}\left(\zeta^{\prime}\right) \tilde{g}\left(\zeta^{\prime \prime}\right) T_{\zeta^{\prime}}^{\hbar} T_{\zeta^{\prime \prime}}^{\hbar} d \zeta^{\prime} d \zeta^{\prime \prime}=\mathfrak{f} \mathfrak{g} .
\end{aligned}
$$

В соответствии с формулами раздела 1 при обратном преобразовании Фурье скрученная свертка (33) переходит в мойаловское произведение

$$
\begin{aligned}
\left(f \star_{\hbar} g\right)(z) & =\frac{1}{(2 \pi)^{2 n}} \int f\left(z+z^{\prime}\right) g\left(z+\frac{\hbar}{2} J t\right) e^{-i z^{\prime} \cdot t} d z^{\prime} d t= \\
& =\frac{1}{(\pi \hbar)^{2 n}} \int f\left(z+z^{\prime}\right) g\left(z+z^{\prime \prime}\right) e^{(2 i / \hbar) z^{\prime} \cdot J z^{\prime \prime}} d z^{\prime} d z^{\prime \prime}
\end{aligned}
$$

В частности, мы видим, что соответствие Вейля есть гомоморфизм из алгебры $\left(S, \star_{\hbar}\right)$ Мойала-Шварца в алгебру ограниченных (более того, ядерных) операторов на $L^{2}\left(\mathbb{R}^{n}, d q\right)$. Подробности обобщения соответствия Вейля на распределения умеренного роста излагаются, например, в работах [4], [20]. Наша цель состоит в распространении его на более широкие классы обобщенных функций.

\section{6. РАСШИРЕНИЕ СООТВЕТСТВИЯ ВЕЙЛЯ}

В основе распространения соответствия Вейля на распределения Шварца лежит тот простой факт, что преобразование Вигнера-Мойала любой пары функций $\varphi, \psi$ из $S\left(\mathbb{R}^{n}\right)$ принадлежит пространству $S\left(\mathbb{R}^{2 n}\right)$ и непрерывно зависит от $\varphi$ и $\psi$. Действительно, по определению (31) функция $W_{\hbar}(\varphi, \psi)$ получается из $\varphi \otimes \psi \in S\left(\mathbb{R}^{2 n}\right)$ линейной заменой переменных и частичным преобразованием Фурье, которые являются автоморфизмами $S\left(\mathbb{R}^{2 n}\right)$. Поэтому правая часть равенства (30) корректно определена для любого распределения $f \in S^{\prime}\left(\mathbb{R}^{2 n}\right)$, если интеграл понимать как его значение на функции $W_{\hbar}(\varphi, \psi)$. Отображение $\varphi \rightarrow\left\langle f, W_{\hbar}(\varphi, \psi)\right\rangle$ линейно и непрерывно, т. е. оно является элементом пространства $S^{\prime}\left(\mathbb{R}^{n}\right)$, причем его зависимость от $\psi \in S\left(\mathbb{R}^{n}\right)$ также линейна и непрерывна. Таким образом, любое распределение $f$ на $\mathbb{R}^{2 n}$ порождает линейный оператор из $S\left(\mathbb{R}^{n}\right)$ в $S^{\prime}\left(\mathbb{R}^{n}\right)$. Если область значений этого оператора содержится в $L^{2}\left(\mathbb{R}^{n}\right) \subset S^{\prime}\left(\mathbb{R}^{n}\right)$, то он становится оператором в $L^{2}\left(\mathbb{R}^{n}\right)$ с областью определения $S\left(\mathbb{R}^{n}\right)$, а распределение $f$ служит его вейлевским символом. Существенно, что соответствие между элементами $S^{\prime}\left(\mathbb{R}^{2 n}\right)$ и операторами из $S\left(\mathbb{R}^{n}\right)$ в $S^{\prime}\left(\mathbb{R}^{n}\right)$ есть биекция согласно теореме Шварца о ядре. Поэтому при обобщении соответствия Вейля мы будем считать пространство пробных функций ядерным. Кроме того, мы ограничимся теми пространствами, для которых каждый раздельно непрерывный билинейный функционал является непрерывным. Хорошо известно, что это так для пространств Фреше и для сопряженных им пространств, 
снабженных сильной топологией. Для ядерных пространств этих двух классов используются аббревиатуры FN и DFN соответственно. Все они являются монтелевскими пространствами. Кроме того, если $E$ является FN- или DFN-пространством, то таково и $E \widetilde{\otimes} E$.

Легко видеть, что существует единственный линейный автогомеоморфизм ${ }^{2)} \mathcal{W}_{\hbar}$ пространства $S\left(\mathbb{R}^{2 n}\right)$ такой, что

$$
\mathcal{W}_{\hbar}(\varphi \otimes \psi)=W_{\hbar}\left(\varphi^{*}, \psi\right) \quad \text { при всех } \quad \varphi, \psi \in S\left(\mathbb{R}^{n}\right) .
$$

Действительно, пусть $\chi \in S\left(\mathbb{R}^{2 n}\right)$. Положим

$$
\mathcal{W}_{\hbar}(\chi)(q, p)=\frac{1}{(2 \pi \hbar)^{n}} \int \chi\left(q-\frac{1}{2} \xi, q+\frac{1}{2} \xi\right) e^{-(i / \hbar) p \xi} d \xi
$$

Тогда условие (34) выполнено и отображение $\chi \rightarrow W_{\hbar}(\chi)$, очевидно, является автогомеоморфизмом $S\left(\mathbb{R}^{2 n}\right)$. Других линейных непрерывных автоморфизмов пространства $S\left(\mathbb{R}^{2 n}\right)$ со свойством $(34)$ не существует, поскольку $S\left(\mathbb{R}^{n}\right) \otimes S\left(\mathbb{R}^{n}\right)$ плотно в нем. Линейное преобразование $\mathcal{W}_{\hbar}$ иногда называют [4] расширенным преобразованием Вигнера-Мойала.

Для любой пары $E, G$ локально выпуклых пространств мы обозначаем через $\mathcal{L}(E, G)$ пространство непрерывных линейных отображений из $E$ в $G$, снабженное топологией ограниченной сходимости. Если $E$ - локально выпуклое подпространство в $S\left(\mathbb{R}^{2 n}\right)$, то его образ при отображении $\mathcal{W}_{\hbar}$ обозначаем через $\mathcal{W}_{\hbar}(E)$ и снабжаем топологией, индуцированной из $E$.

Tеорема 2. Пусть $E$ - произвольное плотное FN- или DFN-nодпространство в $S\left(\mathbb{R}^{n}\right)$ с непрерьвной инволюиией $\varphi \rightarrow \varphi^{*}$ и пусть $\mathcal{E}=\mathcal{W}_{\hbar}(E \widetilde{\otimes} E)$. Соответствие Вейля может быть единственным образом продолжено до непрерывного взаимно однозначного отображения пространства обобщенных функций $\mathcal{E}^{\prime}$ на пространство операторов $\mathcal{L}\left(E, E^{\prime}\right)$. Если образ оператора $\mathfrak{u}$, соответствующего $u \in \mathcal{E}^{\prime}$ при этом отображении, содержится в $L^{2}\left(\mathbb{R}^{n}\right)$, то оператор и непреръвен из $E$ в $L^{2}\left(\mathbb{R}^{n}\right)$.

ДокАЗАТЕльство. В соответствии со сказанным выше для любого функционала $u \in \mathcal{E}^{\prime}$ мы определяем линейный оператор $\mathfrak{u}: E \rightarrow E^{\prime}$, полагая

$$
\langle\mathfrak{u} \psi, \varphi\rangle \stackrel{\text { def }}{=}\left\langle u, W_{\hbar}\left(\varphi^{*}, \psi\right)\right\rangle \equiv\left\langle u, \mathcal{W}_{\hbar}(\varphi \otimes \psi)\right\rangle, \quad \psi, \varphi \in E .
$$

Легко видеть, что оператор $\mathfrak{u}$ непрерывен. Поскольку $E$ - борнологическое пространство, достаточно показать, что он секвенциально непрерывен. Если $\psi_{\nu} \rightarrow 0$ в $E$, то, очевидно, $\left\langle\mathfrak{u} \psi_{\nu}, \varphi\right\rangle \rightarrow 0$ при любой функции $\varphi \in E$. Отсюда следует, что $u_{\psi_{\nu}} \rightarrow 0$ в сильной топологии $E^{\prime}$, поскольку $E$ является монтелевским пространством. Теперь покажем, что отображение

$$
\mathcal{E}^{\prime} \rightarrow L\left(E, E^{\prime}\right): u \rightarrow \mathfrak{u}
$$

2) Автогомеоморфизмом (или топологическим автоморфизмом) называют взаимно однозначное и взаимно непрерывное отображение топологического пространства на себя. 
является непрерывным. Фундаментальную систему окрестностей нуля в $L\left(E, E^{\prime}\right)$ образуют множества вида $\mathcal{U}_{Q}=\left\{\mathfrak{u}: \mathfrak{u}(Q) \subset U_{Q}\right\}$, где $Q$ пробегает ограниченные множества в $E$, а $U_{Q}$ - окрестность нуля в $E^{\prime}$, состоящая из тех $u \in E^{\prime}$, для которых $|\langle u, \varphi\rangle|<1$ при всех $\varphi \in Q$. Множество $\mathcal{Q}=\left\{\chi: \chi=\mathcal{W}_{\hbar}(\varphi \otimes \psi), \varphi, \psi \in Q\right\}$ ограничено в $\mathcal{E}$, поскольку каноническое билинейное отображение $(\varphi, \psi) \rightarrow \varphi \otimes \psi$ из $E \times E$ в $E \widetilde{\otimes} E$ и линейное отображение $\mathcal{W}_{\hbar}$ непрерывны. Если $u$ принадлежит окрестности нуля в $E^{\prime}$, задаваемой условием $\sup _{\mathcal{Q}}|\langle u, \varphi\rangle|<1$, то из определения (36) следует, что $\mathfrak{u} \in \mathcal{U}_{Q}$. Тем самым отображение $u \rightarrow \mathfrak{u}$ непрерывно. Ясно также, что оно инъективно и совпадает с соответствием Вейля на регулярных обобщенных функциях, порождаемых функциями $f \in S\left(\mathbb{R}^{2 n}\right)$, поскольку замена $u \rightarrow f, \varphi \rightarrow \varphi^{*}$ превращает (36) в (30) и поскольку $E$ плотно в $S\left(\mathbb{R}^{n}\right)$, а значит, и в $L^{2}\left(\mathbb{R}^{n}\right)$. Других продолжений соответствия Вейля до непрерывного отображения $\mathcal{E}^{\prime} \rightarrow \mathcal{L}\left(E, E^{\prime}\right)$ не существует, поскольку $S\left(\mathbb{R}^{2 n}\right)$ плотно в $\mathcal{E}^{\prime}$.

Пусть теперь $\mathfrak{u}-$ произвольный оператор из $\mathcal{L}\left(E, E^{\prime}\right)$. Тогда билинейная форма $\langle\mathfrak{u} \psi, \varphi\rangle$ на $E \times E$ раздельно непрерывна. В силу наших предположений о пространстве $E$ она также совместно непрерывна, и, значит, существует единственный линейный функционал $v \in(E \widetilde{\otimes} E)^{\prime}$ такой, что $\langle\mathfrak{u} \psi, \varphi\rangle=\langle v, \varphi \otimes \psi\rangle$ при всех $\varphi, \psi \in E$. В свою очередь, $v$ однозначно определяет функционал $u \in \mathcal{E}^{\prime}$ такой, что

$$
\left\langle u, \mathcal{W}_{h}(\chi)\right\rangle=\langle v, \chi\rangle \quad \text { при всех } \quad \chi \in E \widetilde{\otimes} E,
$$

поскольку $\mathcal{W}_{h}$ есть изоморфизм между $E \widetilde{\otimes} E$ и $\mathcal{E}$. Таким образом, отображение $(37)$ биективно.

Предположим, что область значений оператора $\mathfrak{u}$, определяемого формулой (36), содержится в $L^{2}\left(\mathbb{R}^{n}\right) \subset E^{\prime}$. Тогда он может рассматриваться как оператор в гильбертовом пространстве $L^{2}\left(\mathbb{R}^{n}\right)$ с областью определения $E$ и, очевидно, имеет замкнутый график по отношению к топологии $E$. Следовательно, в этом случае он принадлежит пространству линейных непрерывных отображений $\mathcal{L}\left(E, L^{2}\left(\mathbb{R}^{n}\right)\right)$ в силу теоремы о замкнутом графике в форме Птака ([19], § IV.8.5), которая применима к пространствам рассматриваемого класса. Это завершает доказательство.

Таким образом, вейлевский символ любого оператора из $\mathcal{L}\left(E, L^{2}\right)$ содержится в пространстве обобщенных функций $\left(\mathcal{W}_{\hbar}(E \widetilde{\otimes} E)\right)^{\prime}$. В это пространство входят, конечно, и вейлевские символы, определяемые в рамках теории распределений умеренного роста [4], [20], которые соответствуют операторам из $\mathcal{L}\left(S, L^{2}\right)$.

СлеДСТвИЕ 1. Если для пространства Е выполнены условия теоремы 2 и про-

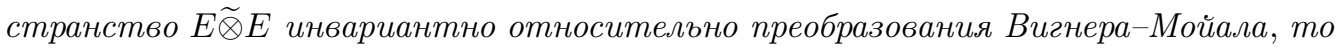
соответствие Вейля распространяется до непрерывной биекции между $(E \widetilde{\otimes} E)^{\prime}$ $u \mathcal{L}\left(E, E^{\prime}\right)$.

В качестве примера рассмотрим пространства $S_{\beta}^{\beta}$. Напомним, что $S_{\beta}^{\beta}\left(\mathbb{R}^{n}\right)$ состоит из тех функций $f(x) \in S\left(\mathbb{R}^{n}\right)$, которые удовлетворяют неравенствам

$$
\left|\partial^{\kappa} f(x)\right| \leqslant C B^{|\kappa|} \kappa^{\beta \kappa} e^{-|x / B|^{1 / \beta}},
$$

где константы $C$ и $B$ зависят от $f$ и использованы обычные мультииндексные обозначения, в частности $\partial^{\kappa}=\partial_{1}^{\kappa_{1}} \ldots \partial_{1}^{\kappa_{d}}, \partial_{j}=\partial / \partial x_{j}, 1 \leqslant j \leqslant n$. Мы будем также 
использовать для этого пространства более краткое обозначение $S_{\beta}^{\beta}$, когда это не может привести к недоразумениям. Оно является объединением банаховых пространств $S_{\beta, B}^{\beta, B}$ с нормами

$$
\|f\|_{B}=\sup _{x, \kappa} e^{|x / B|^{1 / \beta}}\left|\frac{\partial^{\kappa} f(x)}{B^{|\kappa|} \kappa^{\kappa \beta}}\right|,
$$

и его естественная топология есть топология индуктивного предела относительно вложений $S_{\beta, B}^{\beta, B} \rightarrow S_{\beta}^{\beta}$. При снабжении этой топологией $S_{\beta}^{\beta}$ становится DFN-пространством. Оно нетривиально только при $\beta \geqslant 1 / 2$. В монографии [12] доказано, что все пространства $S_{\beta}^{\beta}$ являются алгебрами относительно поточечного умножения и относительно обычной свертки, а в работе [11] доказано, что $S_{\beta}^{\beta}\left(\mathbb{R}^{2 n}\right)$ являются топологическими алгебрами относительно произведения Мойала и скрученной свертки.

ПредлОЖенИЕ 4. Группа Вейля-Гейзенберга непрерьвно действует на $S_{\beta}^{\beta}\left(\mathbb{R}^{n}\right)$, a преобразование Вигнера-Мойала $\mathcal{W}_{\hbar}$ является автогомеоморбизмом пространства $S_{\beta}^{\beta}\left(\mathbb{R}^{2 n}\right)$. При любых $\varphi, \psi \in S_{\beta}^{\beta}\left(\mathbb{R}^{n}\right)$ матричный элемент $\left(\varphi, T_{\zeta}^{\hbar} \psi\right)$ принадлежит пространству $S_{\beta}^{\beta}\left(\mathbb{R}^{2 n}\right)$ и совместно непреръвен по $\varphi, \psi$.

ДокАЗАтельство. В книге [12] доказано, что сдвиги координат и преобразование Фурье являются топологическими изоморфизмами пространств $S_{\beta}^{\beta}$. Нетрудно также проверить, что они инвариантны относительно линейных замен переменных и эти преобразования непрерывны по топологии $S_{\beta}^{\beta}$. Отсюда следуют утверждения относительно группы Вейля-Гейзенберга и преобразования Вигнера-Мойала. Утверждение относительно функции $\left(\varphi, T_{\zeta}^{\hbar} \psi\right)$ столь же очевидно, поскольку она связана с $\mathcal{W}_{\hbar}(\varphi \otimes \psi)(z)$ симплектическим преобразованием Фурье.

ПрЕДЛОЖЕНИЕ 5. Определяемое теоремой 2 расширение соответствия Вейля устанавливает, в частности, взаимно однозначное соответствие между обобщенными функииями над пространством $S_{\beta}^{\beta}\left(\mathbb{R}^{2 n}\right), \beta \geqslant 1 / 2$, и непрерывными операторами из $\mathcal{S}_{\beta}^{\beta}\left(\mathbb{R}^{n}\right)$ в $\left(S_{\beta}^{\beta}\left(\mathbb{R}^{n}\right)\right)^{\prime}$, а каждый оператор, действующий в $L^{2}\left(\mathbb{R}^{n}\right)$ и принадлежащий $\mathcal{L}\left(S_{\beta}^{\beta}\left(\mathbb{R}^{n}\right), L^{2}\left(\mathbb{R}^{n}\right)\right)$, имеет вейлевский символ в $\left(S_{\beta}^{\beta}\left(\mathbb{R}^{2 n}\right)\right)^{\prime}$.

ДокАзАтЕльство. Утверждение немедленно следует из теоремы 2 и соотношения

$$
S_{\beta}^{\beta}\left(\mathbb{R}^{n}\right) \widetilde{\otimes} S_{\beta}^{\beta}\left(\mathbb{R}^{n}\right)=S_{\beta}^{\beta}\left(\mathbb{R}^{2 n}\right),
$$

которое установлено в [21] и выражает аналог теоремы Шварца о ядре для $S_{\beta}^{\beta}$.

Ясно, что пространство вейлевских символов тем больше, чем меньше $\beta$, и при $\beta=1 / 2$ мы получаем максимальное расширение соответствия Вейля. Простейшим примером быстрорастущих функций, которые охватываются теоремой 2, служат экспоненты $e^{s q+r p}$, где $s$ и $r$ вещественны, а $(p, q)$ - координаты стандартного симплектического пространства $\mathbb{R}^{2 n}$, как и в разделе 5 . Они интегрируемы с пробными функциями из любого пространства $S_{\beta}^{\beta}\left(\mathbb{R}^{2 n}\right)$ с $\beta<1$ и являются его мультипликаторами как относительно обычного, так и относительно мойаловского произведения. Соответствующие им операторы в $L^{2}$ с областью определения $S_{\beta}^{\beta}\left(\mathbb{R}^{n}\right)$ записываются, очевидно, как $e^{-(i \hbar / 2) s r} e^{s q} e^{-i \hbar r \partial}$. Более интересным примером является функция 
$u_{a}(q, p)=e^{2 i a q p}$ с вещественным $a$. Хорошо известно [4], что ее преобразование Вейля определено и ограничено на $L^{2}\left(\mathbb{R}^{n}\right)$ в том и только том случае, если $|a| \neq 1 / \hbar$. Действительно, пусть $\varphi, \psi \in S\left(\mathbb{R}^{n}\right)$. Согласно (31) и (36) мы имеем

$$
\begin{aligned}
\left\langle\mathfrak{u}_{a} \psi, \varphi\right\rangle & =\frac{1}{(2 \pi \hbar)^{n}} \int \varphi\left(q-\frac{1}{2} x\right) \psi\left(q+\frac{1}{2} x\right) e^{-(i / \hbar) p x+2 i a q p} d x d q d p= \\
& =\frac{1}{(2 \pi \hbar)^{n}} \int \varphi(x) \psi(y) e^{(i / \hbar) p(x-y)+i a p(x+y)} d x d y d p= \\
& =\int \varphi(x) \psi(y) \delta(x-y+a \hbar(x+y)) d x d y= \\
& =\frac{1}{|1-\hbar a|^{n}} \int \varphi(x) \psi\left(\frac{1+\hbar a}{1-\hbar a} x\right) d x .
\end{aligned}
$$

Следовательно, если $|a| \neq 1 / \hbar$, то

$$
\left(\mathfrak{u}_{a} \psi\right)(x)=\frac{1}{|1-\hbar a|^{n}} \psi\left(\frac{1+\hbar a}{1-\hbar a} x\right) .
$$

При $a=1 / \hbar$ мы имеем

$$
\left\langle\mathfrak{u}_{1 / \hbar} \psi, \varphi\right\rangle=\int \varphi(x) \psi(y) \delta(2 x) d x d y=2^{-n} \varphi(0) \int \psi(y) d y,
$$

и тем самым оператор $\mathfrak{u}_{1 / \hbar}$ переводит функцию $\psi$ в распределение

$$
2^{-n}\left(\int \psi(y) d y\right) \delta(x)
$$

Если $a=-1 / \hbar$, то

$$
\left\langle\mathfrak{u}_{-1 / \hbar} \psi, \varphi\right\rangle=\int \varphi(x) \psi(y) \delta(2 y) d x d y=2^{-n} \psi(0) \int \varphi(x) d x,
$$

и мы видим, что $\mathfrak{u}_{-1 / \hbar} \psi=2^{-n} \psi(0)$.

Этот пример дает полезную информацию об алгебре мойаловских мультипликаторов $\mathcal{M}_{\theta}\left(S_{\beta}^{\beta}\right)$, соответствующей $\theta=\hbar J$. Мы будем обозначать ее через $\mathcal{M}_{\hbar}\left(S_{\beta}^{\beta}\right)$, а алгебру поточечных мультипликаторов этого пространства - через $M\left(S_{\beta}^{\beta}\right)$.

ПРЕДЛОЖЕНИЕ 6. Пусть $(q, p)$ - координаты стандартного симплектического пространства $\mathbb{R}^{2 n}$ и $а \in \mathbb{R}$. Функиия $e^{2 і а q р ~ п р и н а д л е ж и т ~ а л г е б р е ~} \mathcal{M}_{\hbar}\left(S_{\beta}^{\beta}\right)$ в том и только том случае, если $|a| \neq 1 / \hbar$.

ДокАЗАтЕЛЬство. Воспользуемся формулой

$$
f \star_{\hbar} u=\left(\frac{2}{\hbar}\right)^{2 n} \tilde{f}_{2 / \hbar} \circledast 4 / \hbar u,
$$

где

$$
\tilde{f}_{2 / \hbar}(z):=\tilde{f}(2 z / \hbar)=\frac{1}{(2 \pi)^{n}} \int f\left(z^{\prime}\right) e^{-(2 i / \hbar) z^{\prime} \cdot J z} d z^{\prime}
$$


Если $f, u \in S$, то формула (41) прямо следует из (2), (4) при $\theta=\hbar J$, а на распределения $u \in S^{\prime}$ она распространяется по непрерывности (подробнее см. [17]). При

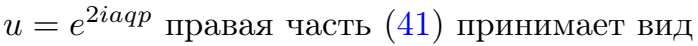

$$
\begin{aligned}
\left(\frac{2}{\hbar}\right)^{2 n} & \frac{1}{(2 \pi)^{n}} \int \tilde{f}_{2 / \hbar}\left(q^{\prime}, p^{\prime}\right) e^{2 i a\left(q-q^{\prime}\right)\left(p-p^{\prime}\right)} e^{(2 i / \hbar)\left(q p^{\prime}-p q^{\prime}\right)} d q^{\prime} d p^{\prime}= \\
= & \frac{1}{(2 \pi)^{n}} e^{2 i a q p} \int \tilde{f}(\xi, \eta) e^{\left(i \hbar^{2} a / 2\right) \xi \eta} e^{i(1-\hbar a) q \eta-i(1+\hbar a) p \xi} d \xi d \eta
\end{aligned}
$$

Пусть $f \in S_{\beta}^{\beta}\left(\mathbb{R}^{2 n}\right)$. Поскольку пространство $S_{\beta}^{\beta}$ инвариантно относительно преобразования Фурье и растяжений координат, а $e^{\text {iaqp }}$ является для него поточечным мультипликатором, мы видим, что функция (42) принадлежит $S_{\beta}^{\beta}\left(\mathbb{R}^{2 n}\right)$, если $|a| \neq 1 / \hbar$. Следовательно, в этом случае $e^{i a q p} \in \mathcal{M}_{\hbar, R}\left(S_{\beta}^{\beta}\right)$. Аналогичная выкладка с использованием формулы $u \star_{\hbar} f=(2 / \hbar)^{2 n} u \circledast_{4 / \hbar} \tilde{f}_{-2 / \hbar}$ дает $e^{i a q p} \in \mathcal{M}_{\hbar, L}$. Если $|a|=1 / \hbar$, то интеграл в правой части (42) зависит лишь от одной из переменных $q$ или $p$, и эта функция, очевидно, не принадлежит $S_{\beta}^{\beta}\left(\mathbb{R}^{2 n}\right)$ ни при каком $f \neq 0$. Предложение доказано.

СлеДСТВИЕ 2. Алгебры $\mathcal{M}_{\hbar}\left(S_{\beta}^{\beta}\right)$ с различными значениями параметра $\hbar>0$ все различаются между собой и $M\left(S_{\beta}^{\beta}\right) \nsubseteq \mathcal{M}_{\hbar}\left(S_{\beta}^{\beta}\right)$ при любом $\hbar \neq 0$.

Это обобщает известный результат [15] для $\mathcal{M}_{\hbar}(S)$. Предложение 6 допускает дальнейшее обобщение на алгебру $\mathcal{M}_{\hbar}(E)$ для любого удовлетворяющего условиям теоремы 1 и инвариантного относительно преобразования Фурье пространства $E$. Отметим еще, что при $\beta<\alpha$ алгебра $\mathcal{M}_{\hbar}\left(S_{\alpha}^{\beta}\right)$ не содержит $e^{\text {iaqp }}$ ни при каком вещественном $a \neq 0$.

\section{7. ПРЕОБРАЗОВАНИЕ ВЕЙЛЯ МОЙАЛОВСКИХ МУЛЬТИПЛИКАТОРОВ}

Если $E$ - топологическое векторное пространство функций на $\mathbb{R}^{n}$, на котором непрерывно действует группа Вейля-Гейзенберга, то для любых $v \in E^{\prime}$ и $\varphi \in E$ определена и непрерывна функция

$$
f_{v, \varphi}(\zeta)=\left\langle v, T_{\zeta}^{\hbar} \varphi\right\rangle
$$

Нам понадобится следующее добавление к предложению 4.

ПреДЛОЖЕНИЕ 7. Пусть $v \in\left(S_{\beta}^{\beta}\left(\mathbb{R}^{n}\right)\right)^{\prime}, \beta \geqslant 1 / 2$. Если при всех $\varphi \in S_{\beta}^{\beta}\left(\mathbb{R}^{n}\right)$ функиия (43) принадлежит пространству $S_{\beta}^{\beta}\left(\mathbb{R}^{2 n}\right)$, то функционал $v$ порождается функиией из $S_{\beta}^{\beta}\left(\mathbb{R}^{n}\right)$.

ДокАЗАТЕльство. Поскольку пространства $S_{\beta}^{\beta}$ инвариантны относительно растяжений координат, можно считать, что $\hbar=1$. Тогда функция (43) записывается каK

$$
f_{v, \varphi}(\xi, \eta)=e^{-(i / 2) \xi \eta}\left\langle v, e^{i(\eta, \cdot)} \varphi(\cdot-\xi)\right\rangle
$$


Полагая $\xi=0$ и учитывая, что ограничение любой функции из $S_{\beta}^{\beta}\left(\mathbb{R}^{2 n}\right)$ на плоскость $\left\{(\xi, \eta) \in \mathbb{R}^{2 n}: \xi=0\right\}$ принадлежит $S_{\beta}^{\beta}\left(\mathbb{R}^{n}\right)$, мы получаем

$$
\left\langle v, e^{i(\eta, \cdot)} \varphi\right\rangle=(\hat{v}, \hat{\varphi}(-\eta-\cdot))=(\hat{v} * \hat{\varphi})(-\eta) \in S_{\beta}^{\beta}\left(\mathbb{R}^{n}\right) \quad \text { при всех } \quad \varphi \in S_{\beta}^{\beta}\left(\mathbb{R}^{n}\right) .
$$

Поскольку пространство $S_{\beta}^{\beta}$ инвариантно относительно отражений и к нему применима теорема о замкнутом графике, мы видим, что $\hat{v}$ является для него свертывателем, а $v$ - мультипликатором относительно поточечного умножения. Легко проверяется, что пространство $S_{\beta}^{\beta}$, рассматриваемое как алгебра относительно поточечного умножения, имеет аппроксимативную единицу ${ }^{3)}$. Поэтому порождаемый мультипликатором $v$ оператор $V \in \mathcal{L}\left(S_{\beta}^{\beta}\right)$ допускает представление в виде предела операторов умножения на функции $v_{\nu} \in S_{\beta}^{\beta}$. В частности, $v_{\nu} g \rightarrow V g$ в $S_{\beta}^{\beta}$ при любой функции $g \in S_{\beta}^{\beta}$. Поскольку топология $S_{\beta}^{\beta}$ сильнее топологии поточечной сходимости и для любой точки $q_{0} \in \mathbb{R}^{n}$ найдется $g \in S_{\beta}^{\beta}$ такая, что $g\left(q_{0}\right) \neq 0$, мы заключаем, что последовательность $v_{\nu}$ поточечно сходится к некоторой непрерывной (и даже гладкой) функции $v(q)$, причем $v(q) g(q)=(V g)(q)$ при всех $g \in S_{\beta}^{\beta}, q \in \mathbb{R}^{n}$. Функционал $v$, очевидно, порождается этой функцией, поскольку $\langle v, g\rangle \equiv \int(V g)(q) d q$. При любом фиксированном $\xi$ произведение $v(q) \varphi(q-\xi)$, рассматриваемое как функция переменной $q$, принадлежит $S_{\beta}^{\beta}\left(\mathbb{R}^{n}\right)$, и из (44) следует, что

$$
v(q) \varphi(q-\xi)=\frac{1}{(2 \pi)^{n}} \int e^{-i \eta q} e^{(i / 2) \xi \eta} f_{v, \varphi}(\xi, \eta) d \eta
$$

Выбрав $\varphi$ так, чтобы $\varphi(0)=1$, мы получаем

$$
v(q)=\left.\frac{1}{(2 \pi)^{n}} \int e^{-(i / 2) \xi \eta} f_{v, \varphi}(q, \eta) d \eta\right|_{\xi=q} .
$$

Значит, $v \in S_{\beta}^{\beta}\left(\mathbb{R}^{n}\right)$, поскольку частичное преобразование Фурье есть автоморфизм $S_{\beta}^{\beta}\left(\mathbb{R}^{2 n}\right)$, а ограничение на диагональ $\xi=q$ дает элемент в $S_{\beta}^{\beta}\left(\mathbb{R}^{n}\right)$. Предложение доказано.

Теорема 3. Определенное теоремой 2 соответствие Вейля устанавливает, в частности, изоморфизм между алгеброй $\mathcal{M}_{h, L}\left(S_{\beta}^{\beta}\right)$ левых мойаловских мультипликаторов пространства $S_{\beta}^{\beta}\left(\mathbb{R}^{2 n}\right)$ и алгеброй $\mathcal{L}\left(S_{\beta}^{\beta}\right)$ операторов в $L^{2}\left(\mathbb{R}^{n}\right)$, непрерывно отображающих пространство $S_{\beta}^{\beta}\left(\mathbb{R}^{n}\right)$ в себя.

ДоказАтельство. Пусть $u \in \mathcal{M}_{h, L}\left(S_{\beta}^{\beta}\right)$ и оператор $\mathfrak{u}: S_{\beta}^{\beta}\left(\mathbb{R}^{n}\right) \rightarrow\left(S_{\beta}^{\beta}\left(\mathbb{R}^{n}\right)\right)^{\prime}$ определен формулой (36). Покажем, что и отображает $S_{\beta}^{\beta}\left(\mathbb{R}^{n}\right)$ в себя. Для этого применим предложение 7 , заметив предварительно, что сформулированное в нем условие равносильно тому, что функция $\left\langle v,\left(T_{\zeta}^{\hbar} \varphi\right)^{*}\right\rangle$ принадлежит $S_{\beta}^{\beta}\left(\mathbb{R}^{2 n}\right)$ при всех $\varphi \in S_{\beta}^{\beta}\left(\mathbb{R}^{n}\right)$. Действительно, $\left(T_{\zeta}^{\hbar} \varphi\right)^{*}=T_{\bar{\zeta}}^{\hbar} \varphi^{*}$, где $\bar{\zeta}=(\xi,-\eta)$, а пространства $S_{\beta}^{\beta}$ инвариантны относительно комплексного сопряжения и относительно отражения

3)Это следует и из доказанного в работе [16] существования аппроксимативной единицы в $\left(S_{\alpha}^{\beta}, \star_{\theta}\right)$ при любом $\theta$. 
любой из координат. Мы воспользуемся еще тем, что скрученная свертка функции $g \in S_{\beta}^{\beta}\left(\mathbb{R}^{2 n}\right)$ с любым функционалом $w$ из сопряженного пространства может быть записана как

$$
(w \circledast \hbar g)(s)=\frac{1}{(2 \pi)^{n}}\left\langle w, e^{(i \hbar / 2) s \cdot J(\cdot)} g(s-\cdot)\right\rangle .
$$

Справедливость этого представления устанавливается теоремой 5 в работе [16]. Рассмотрим функцию $\chi(\zeta)=\left\langle\mathfrak{u} \psi,\left(T_{\zeta}^{\hbar} \varphi\right)^{*}\right\rangle$, где $\varphi, \psi \in S_{\beta}^{\beta}\left(\mathbb{R}^{n}\right)$. Поскольку симплектические преобразования Фурье $\mathcal{F}_{J}$ и $\overline{\mathcal{F}}_{J}$ сопряжены друг другу относительно спаривания $\langle\cdot, \cdot\rangle$, из $(36)$ и $(32)$ немедленно следует

$$
\chi(\zeta)=\left\langle u, W_{\hbar}\left(T_{\zeta}^{\hbar} \varphi, \psi\right)\right\rangle=\frac{1}{(2 \pi)^{n}}\left\langle\tilde{u},\left(T_{\zeta}^{\hbar} \varphi, T_{(\cdot)}^{\hbar} \psi\right)\right\rangle .
$$

Из соотношения (27) вытекает, что

$$
\left(T_{\zeta}^{\hbar} \varphi, T_{\zeta^{\prime}}^{\hbar} \psi\right)=\left(T_{-\zeta^{\prime}}^{\hbar} T_{\zeta}^{\hbar} \varphi, \psi\right)=e^{(i \hbar / 2) \zeta \cdot J \zeta^{\prime}}\left(T_{\zeta-\zeta^{\prime}}^{\hbar} \varphi, \psi\right) .
$$

Вводя обозначение $g(\zeta)=\left(T_{\zeta}^{\hbar} \varphi, \psi\right)=\left(\varphi, T_{-\zeta}^{\hbar} \psi\right)$ и используя (45), мы получаем $\chi=$ $\tilde{u} \circledast \hbar g$, что равносильно равенству

$$
\tilde{\chi}=u \star_{\hbar} \tilde{g},
$$

поскольку $\tilde{\tilde{u}}=u$. Согласно предложению 4 функция $\tilde{g}$ принадлежит фурье-инвариантному пространству $S_{\beta}^{\beta}\left(\mathbb{R}^{2 n}\right)$, поэтому мойаловское произведение в правой части (47) также принадлежит ему. Мы заключаем, что $\chi \in S_{\beta}^{\beta}\left(\mathbb{R}^{2 n}\right)$ и в силу предложения $7 \mathfrak{u} \psi \in S_{\beta}^{\beta}\left(\mathbb{R}^{n}\right)$ при всех $\psi \in S_{\beta}^{\beta}\left(\mathbb{R}^{n}\right)$. Итак, оператор и действительно переводит пространство $S_{\beta}^{\beta}\left(\mathbb{R}^{n}\right)$ в себя, а в силу теоремы о замкнутом графике он непрерывен по его топологии, т. е. принадлежит $\mathcal{L}\left(S_{\beta}^{\beta}\right)$. Ясно также, что отображение $\mathcal{M}_{h, L}\left(S_{\beta}^{\beta}\right) \rightarrow \mathcal{L}\left(S_{\beta}^{\beta}\right): u \rightarrow \mathfrak{u}$ инъективно и является гомоморфизмом алгебр.

Обратно, пусть $\mathfrak{u}$ - линейный непрерывный оператор $S_{\beta}^{\beta}\left(\mathbb{R}^{n}\right) \rightarrow S_{\beta}^{\beta}\left(\mathbb{R}^{n}\right)$. По предложению 4 функция

$$
\left(T_{\zeta}^{\hbar} \varphi^{*}, \mathfrak{u} \psi\right)=\left(\varphi^{*}, T_{-\zeta^{\hbar}}^{\hbar} \psi\right)
$$

принадлежит $S_{\beta}^{\beta}\left(\mathbb{R}^{2 n}\right)$ и билинейное отображение $S_{\beta}^{\beta}\left(\mathbb{R}^{n}\right) \times S_{\beta}^{\beta}\left(\mathbb{R}^{n}\right) \rightarrow S_{\beta}^{\beta}\left(\mathbb{R}^{2 n}\right)$, сопоставляющее паре $\varphi, \psi$ эту функцию, совместно непрерывно. В силу теоремы о ядре, выраженной формулой (40), существует единственное линейное непрерывное отображение $U$ пространства $S_{\beta}^{\beta}\left(\mathbb{R}^{2 n}\right)$ в себя такое, что

$$
U(\varphi \otimes \psi)=\left(T_{\zeta}^{\hbar} \varphi^{*}, \mathfrak{u} \psi\right) .
$$

Согласно теореме 2 существует единственная обобщенная функция $u \in\left(S_{\beta}^{\beta}\left(\mathbb{R}^{2 n}\right)\right)^{\prime}$, связанная с оператором $\mathfrak{u}$ формулой (36), где в данном случае

$$
\langle\mathfrak{u} \psi, \varphi\rangle=\int(\mathfrak{u} \psi)(x) \varphi(x) d x=\left(\varphi^{*}, \mathfrak{u} \psi\right) .
$$

Следовательно,

$$
\left(T_{\zeta}^{\hbar} \varphi^{*}, \mathfrak{u} \psi\right)=\left\langle\mathfrak{u} \psi,\left(T_{\zeta}^{\hbar} \varphi^{*}\right)^{*}\right\rangle=\left\langle u, W_{\hbar}\left(T_{\zeta}^{\hbar} \varphi^{*}, \psi\right)\right\rangle .
$$


Выражение в правой части (48) получается из функции (46) заменой $\varphi$ на $\varphi^{*}$ и может быть записано как

$$
\tilde{u} \circledast \hbar g_{1}=\widetilde{u \star \hbar \widetilde{g_{1}}},
$$

где $g_{1}(\zeta)=\left(\varphi^{*}, T_{-\zeta}^{\hbar} \psi\right)$. По формулам (32) и (34) мы имеем $\tilde{g}_{1}=(2 \pi)^{n} W_{\hbar}\left(\varphi^{*}, \psi\right)=$ $(2 \pi)^{n} \mathcal{W}_{\hbar}(\varphi \otimes \psi)$. Поскольку $\mathcal{W}_{\hbar}$ есть топологический изоморфизм пространства $S_{\beta}^{\beta}\left(\mathbb{R}^{2 n}\right)$, отображение

$$
f \rightarrow(2 \pi)^{n} \mathcal{F}_{J}\left(u \star_{\hbar} \mathcal{W}_{\hbar}(f)\right)
$$

из этого пространства в сопряженное ему является непрерывным. Отображение (49) совпадает с $U$ на плотном множестве $S_{\beta}^{\beta}\left(\mathbb{R}^{n}\right) \otimes S_{\beta}^{\beta}\left(\mathbb{R}^{n}\right)$ и, следовательно, всюду. Поэтому его область значений содержится в $S_{\beta}^{\beta}\left(\mathbb{R}^{2 n}\right)$. Тем самым $u \star_{\hbar} \mathcal{W}_{\hbar}(f) \in$ $S_{\beta}^{\beta}\left(\mathbb{R}^{2 n}\right)$ при всех $f \in S_{\beta}^{\beta}\left(\mathbb{R}^{2 n}\right)$ и, значит, $u \in \mathcal{M}_{\hbar, L}\left(S_{\beta}^{\beta}\right)$. Теорема доказана.

СлеДСТвИЕ 3. Обобщенная функиия $u \in\left(S_{\beta}^{\beta}\left(\mathbb{R}^{2 n}\right)\right)^{\prime}$ принадлежит алгебре мультипликаторов $\mathcal{M}_{\hbar}\left(S_{\beta}^{\beta}\right)$ в том и только том случае, если ее преобразование Вейля $\mathfrak{u}$, а также эрмитово сопряженный оператор $\mathfrak{u}^{*}$ непрерывно отображают пространство $S_{\beta}^{\beta}\left(\mathbb{R}^{n}\right)$ в себя.

Действительно, условие $u \in \mathcal{M}_{\hbar, R}\left(S_{\beta}^{\beta}\right)$ равносильно условию $u^{*} \in \mathcal{M}_{\hbar, L}\left(S_{\beta}^{\beta}\right)$, а функция $u^{*}$ служит символом Вейля оператора $\left.\mathfrak{u}^{*}\right|_{S_{\beta}^{\beta}}$.

\section{8. ЗАКЛЮЧЕНИЕ}

Как уже было отмечено в разделе 1 , основной областью приложения полученных результатов может быть квантовая теория поля на некоммутативных пространствах, включая развитие ее аксиоматической формулировки, инициированное работой [7]. В рамках распределений умеренного роста наиболее интересные результаты относительно некоммутативной деформации вайтмановской квантовой теории поля получены в работах [22], [23], где показано, что деформированные поля сохраняют локализуемость в конусоподобных областях, и обнаружены связи с двумерными интегрируемыми моделями. Возможность объединения некоммутативной деформации с идеями нелокальной теории поля, использующей аналитические пробные функции, обсуждалась в работе [24], где показано, что адекватное обобщение условия микропричинности обеспечивает СРТ-инвариантность теории. Пространства типа $S$ Гельфанда-Шилова использовались в ряде работ [25]-[27] при анализе свойств перенормируемости скалярной теории поля на некоммутативном пространстве Минковского. Помимо приложений к квантовой теории поля развитая здесь техника может быть полезна, по мнению автора, в нестандартных задачах квантовой механики, например при изучении моделей с неэрмитовыми РТ-симметричными гамильтонианами. Подход к этим моделям, основанный на использовании произведения Мойала, предлагался в работе [28], однако рассматриваемое там звездочное произведение соответствует $p q-$-упорядочению, а не вейлевскому упорядочению операторов. Отметим в заключение, что мы ограничились в разделе 7 пространствами $S_{\beta}^{\beta}$ главным образом ради наглядности. Доказанные теоремы можно распространить на любое пространство пробных функций, удовлетворяющее условиям теоремы 1 и инвариантное относительно преобразования Фурье и преобразования Вигнера-Мойала, но это требует более специальных сведений из теории топологических векторных пространств. 


\section{ПРИЛОЖЕНИЕ}

Лемма 1. Пусть $A$ - инволютивнал топологическал алгебра функиий на множестве $X$, причем пространство А монтелевское и его топология сильнее топологии простой сходимости. Если последовательность самосопряженных элементов $e_{n} \in A$ такова, что $\left(e_{n} a\right)(x) \rightarrow a(x)$ при всех $a \in A, x \in X$, и если для каждой функиии $а \in A$ множсетво $\left\{e_{n} a: n=1,2, \ldots\right\}$ ограничено в $A$, то $e_{n}$ является аппроксимативной единицей алгебры $A$.

ДокАЗАТЕЛЬСтво. По определению монтелевского пространства [19] ограниченная последовательность $\left\{e_{n} a\right\}$ имеет в $A$ по крайней мере одну предельную точку. При условии, что топология $A$ сильнее топологии простой сходимости, и в силу предельного соотношения $\lim _{n \rightarrow \infty}\left(e_{n} a\right)(x)=a(x)$ предельной точкой может быть только $a$, тем самым $e_{n} a \rightarrow a$ по топологии $A$. Дальнейшая аргументация аналогична используемой при доказательстве предложения 2. Всякое монтелевское пространство бочечно, и к нему применима обобщенная теорема Банаха-Штейнгауза, согласно которой отсюда следует, что последовательность непрерывных линейных отображений $a \rightarrow e_{n} a$ сходится к тождественному отображению $\operatorname{id}_{A}$ равномерно на каждом предкомпактном подмножестве $A$. В монтелевском пространстве любое ограниченное множество предкомпактно, и мы заключаем, что последовательность операторов умножения слева на $e_{n}$ сходится к $\mathrm{id}_{A}$ по топологии ограниченной сходимости в пространстве операторов $\mathcal{L}(A)$. Из непрерывности инволюции в $A$ следует, что последовательность операторов умножения справа на $e_{n}$ также сходится к $\operatorname{id}_{A}$ по топологии $\mathcal{L}(A)$, что завершает доказательство.

Благодарности. Работа поддержана РФФИ (грант № 12-01-00865).

\section{Список литературы}

[1] Г. Вейль, Теория групп и квантовая механика, Наука, М., 1986.

[2] J. E. Moyal, Proc. Camb. Phylos. Soc., 45:01 (1949), 99-124.

[3] C.K. Zachos, D. B. Fairlie, T. L. Curtright (eds.), Quantum Mechanics in Phase Space, World Scientific Series in 20th Century Physics, 34, World Scientific, Singapore, 2005.

[4] G. B. Folland, Harmonic Analysis in Phase Space, Annals of Mathematics Studies, 122, Princeton Univ. Press, Princeton, NJ, 1989.

[5] S. Doplicher, K. Fredenhagen, J. E. Roberts, Commun. Math. Phys., 172:1 (1995), 187-220, arXiv: hep-th/0303037.

[6] R. J. Szabo, Phys. Rep., 378:4 (2003), 207-299, arXiv: hep-th/0109162.

[7] L. Álvarez-Gaumé, M. A. Vázquez-Mozo, Nucl. Phys. B, 668:1-2 (2003), 293-321, arXiv: hep-th/0305093.

[8] М. А. Соловьев, ТМФ, 121:1 (1999), 139-164.

[9] Р. Стритер, А. Вайтман, РСТ, спин и статистика и все такое, Наука, М., 1966.

[10] М. А. Соловьев, ТМФ, 153:1 (2007), 3-17.

[11] M. A. Soloviev, J. Phys. A, 40:48 (2007), 14593-14604, arXiv: 0708.1151.

[12] И. М. Гельфанд, Г.Е. Шилов, Обобщенные функции, т. 2: Пространства основных и обобщенных функиий, Физматлит, М., 1958.

[13] M. A. Antonets, Lett. Math. Phys., 2:3 (1978), 241-245.

[14] J. M. Gracia-Bondia, F. Lizzi, G. Marmo, P. Vitale, JHEP, 04 (2002), 026, 36 pp., arXiv: hep-th/0112092. 
[15] V. Gayral, J. M. Gracia-Bondia, B. Iochum, T. Schücker, J. C. Várilly, Commun. Math. Phys., 246:3 (2004), 569-623, arXiv: hep-th/0307241.

[16] M. A. Soloviev, J. Math. Phys., 52:6 (2011), 063502, 18 pp., arXiv: 1012.0669.

[17] М. А. Соловьев, ТМФ, 172:1 (2012), 9-27.

[18] Дж. Мёрфи, $C^{*}$-алгебры и теория операторов, Факториал, М., 1997.

[19] Х. Шефер, Топологические векторные пространства, Мир, М., 1966.

[20] J. M. Maillard, J. Geom. Phys., 3:2 (1986), 231-261.

[21] Б. С. Митягин, Труды ММO, 9 (1960), 317-328.

[22] H. Grosse, G. Lechner, JHEP, 09 (2008), 131, 29 pp., arXiv: 0808.3459.

[23] G. Lechner, Deformations of quantum field theories and integrable models, arXiv: 1104.1948.

[24] М. А. Соловьев, ТМФ, 147:2 (2006), 257-269.

[25] A. Fisher, R. J. Szabo, JHEP, 02 (2009), 031, 37 pp., arXiv: 0810.1195.

[26] J. Zahn, Ann. Henri Poincaré, 12:4 (2011), 777-804, arXiv: 1005.0541.

[27] A. Fisher, R. J. Szabo, Phys. Rev. D, 84:12 (2011), 125010, 29 pp., arXiv: 1106.6166.

[28] F. G. Scholtz, H. B. Geyer, J. Phys. A, 39:32 (2006), 10189-10205, arXiv: quant-ph/0602187. 